\title{
The Safety of Low-Molecular Weight Heparins for the Prevention of Thromboembolic Events after Cardioversion of Atrial Fibrillation
}

\author{
Zerrin Y̌IGIT, ${ }^{1}$ MD, M. Serdar KÜÇÜǨOGLU, ${ }^{1}$ MD, Baris ÖKÇÜN, ${ }^{1}$ MD, \\ Vedat SANSOY, ${ }^{1} \mathrm{MD}$, and Deniz GÜZELSOY, ${ }^{1} \mathrm{MD}$
}

\begin{abstract}
SUMMARY
Transesophageal echocardiography (TEE) guided early cardioversion (CV) in conjunction with short-term anticoagulation has been shown to be safe, and an alternative to prolonged conventional anticoagulation therapy. Recently, low molecular weight heparins (LMWHs) have been used successfully as an alternative to standard heparin therapy obviating the need for hospitalization and APTT monitoring. The aim of this study was to determine the feasibility and safety of TEE guided early cardioversion in conjunction with short-term LMWH use in patients with nonvalvular atrial fibrillation (NVAF).

The study group consisted of 172 consecutive patients with NVAF. Before TEE, 90 patients received LMWH (Dalteparin $2 \times 5,000 \mathrm{U}$ ) and 82 patients received standard heparin (UFH) $(5,000 \mathrm{U}$ bolus followed by infusion to raise APTT to 1.5 times control). TEE was performed and the left atrium and left atrial appendage were examined thoroughly for the presence of thrombus. One patient from each group was excluded due to detection of a left atrial thrombus by TEE. Immediately after TEE, CV was attempted and warfarin was initiated. All patients received warfarin for one month after CV. In the LMWH group, 89 of 90 patients $(98.9 \%$ ) were successfully cardioverted. CV was successful in $97.5 \%$ of the patients in the UFH group. None of the patients experienced thromboembolic events during the four weeks after CV.

TEE guided early CV in conjunction with short-term LMWH treatment is as safe as UFH for the prevention of thromboembolic events after CV. (Jpn Heart J 2003; 44: 369377)
\end{abstract}

Key words: Atrial fibrillation, Low molecular weight heparin, Transesophageal echocardiography, Cardioversion

$\mathrm{IN}_{\mathrm{N}}$ patients with recurrent episodes of self-limited paroxysmal atrial fibrillation (AF), or in whom normal sinus rhythm is to be reestablished chemically or electrically, there is a significant risk of thromboembolism. ${ }^{1)}$ In patients with AF lasting 48 hours or more, oral anticoagulation for a minimum of 3 weeks before and

From ${ }^{1}$ Institute of Cardiology, University of Istanbul, Istanbul, Turkey.

Address for correspondence: Associate Prof. Zerrin Yigit MD, Mazhar bey cad. Çamlik ap. No:81/4, Feneryolu, Kadiköy, Istanbul, Turkey.

This study was presented at the 4th World Stroke Conference in November 25-29, 2000, Melbourne, Australia.

Received for publication July 30, 2002.

Revised and accepted September 6, 2002. 
one month after $\mathrm{CV}$ is recommended. ${ }^{2)}$ Another approach combines transesophageal echocardiography (TEE) and pre-TEE standard unfractionated heparin (UFH) in patients with AF longer than 48 hours, a strategy which reduced the duration of hospitalization and may be cost-effective. ${ }^{3,4)}$ In the absence of intracardiac thrombus, early CV is performed. However, UFH treatment has several limitations; its anticoagulant effect has low bioavailability and high variability necessitating repeated checks of the activated partial thromboplastin time in order to adjust the dosage. ${ }^{5)}$ In addition, UFH treatment carries a risk of thrombocytopenia. ${ }^{6)}$

Low molecular weight heparins (LMWHs) are rapidly emerging as an alternative form of anticoagulant therapy to UFH. They are formed by controlled enzymatic or chemical depolymerization of UFH producing monosaccharide chains of varying lengths ( 3 to $7 \mathrm{kD}$ ) but with a mean molecular weight of approximately $5 \mathrm{kD} .{ }^{7)}$ Similar to UFH, LMWHs exert their anticoagulant activity by activating angiotensin (AT) III. The principal difference between LMWHs and UFH lies in their relative abilities to catalyze inactivation of factor-Xa and factor$\mathrm{IIa}$, which is dependent upon the relative composition of molecules with high affinity to AT III called high-affinity molecules. ${ }^{7)}$

Numerous studies and meta-analysis have shown that LMWHs are at least as effective and safer than UFH in the prevention and treatment of venous thromboembolic events. ${ }^{8,9)}$ The LMWHs represent an interesting therapeutic alternative to UFH since they have a higher bioavailability (>95\%) and a more stable anticoagulant effect which does not require laboratory monitoring. ${ }^{10)}$

The aim of this study was to determine the feasibility and safety of TEE guided early $\mathrm{CV}$ in conjunction with short-term LMWH use in patients with nonvalvular AF.

\section{Patients ANd Methods}

This study was a prospective, randomized, parallel group trial. The primary aim was to compare the difference in the rate of thromboembolic events of TEE guided early CV with short-term LMWH use in patients with nonvalvular persistent AF. From October 1999 through September 2001, we evaluated 172 consecutive adult (age range, 18-80 years) patients with nonvalvular persistent AF for at least 48 hours (mean AF duration was $80.2 \pm 65.3$ days). The study population included 88 men and 84 women aged $62.6 \pm 10.2$ years. The patients eligible for the study were hospitalized 24 hours prior to CV. Before TEE 90 patients received LMWH (Dalteparin $2 \times 5,000 \mathrm{U}$ ) and 82 patients received standard heparin $(5,000 \mathrm{U}$ bolus followed by infusion to raise APTT to 1.5 times control). 
Transthoracic echocardiography (TTE) was performed in all patients during the first 24 hours before cardioversion with a 2.5 to $3.5 \mathrm{mHz}$ probe used with a commercially available device (Acuson Sequoia). The TTE variables evaluated included left ventricular ejection fraction, left ventricle diameter, left atrium diameter, mitral valve regurgitation, mitral valve prolapse, and mitral annular calcification.

TEE was performed with a $7 \mathrm{mHz}$ multiplane probe. Patients received posterior pharyngeal anesthesia with $10 \%$ lidocaine spray after at least 4 hours fasting. Tranquilizing drugs were not administered except when required. TEE was performed and the left atrium and left atrial appendage were examined thoroughly for the presence of thrombus. One patient from each group was excluded due to the detection of left atrial thrombus by TEE. Spontaneous echo contrast was defined as dynamic echoes with a swirling pattern distinct from white noise artifact in the left atrium or left atrium appendage cavity. ${ }^{11)}$

Immediately after TEE, CV was attempted; either medical or electrical CV (Figure) was used.

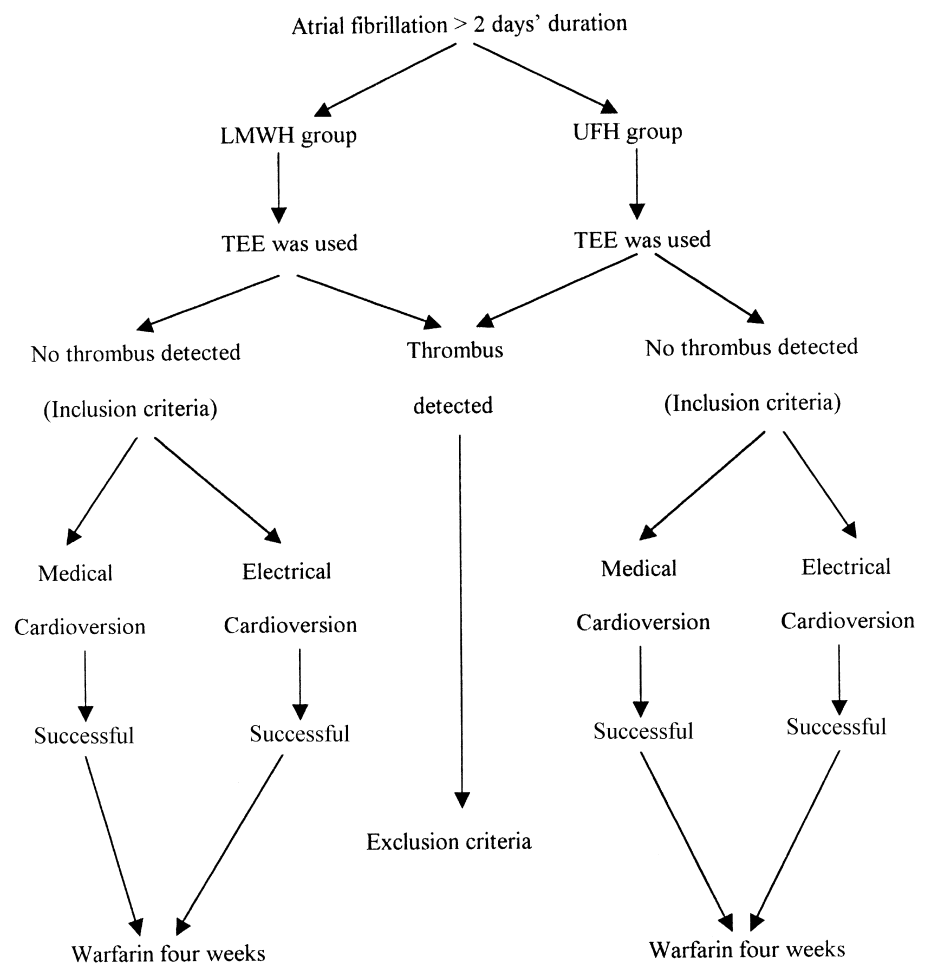

Figure. The study protocol. 
Medical cardioversion: Medical CV was attempted in 86 patients, 83 (96\%) received $800 \mathrm{mg}(4 \times 200 \mathrm{mg})$ quinicardine on the first day, and the dosage was increased $800 \mathrm{mg}$ daily for three days until sinus rhythm was restored. Three patients received an intravenous bolus of $150 \mathrm{mg}$ of amiodarone through a peripheral intravenous line, and amiodarone was continued at $1 \mathrm{mg} / \mathrm{min}$ for 6 hours followed by $0.5 \mathrm{mg} / \mathrm{min}$ for 12 hours. CV was unsuccessful in 3 patients (one in the LMWH group and 2 in the UFH group).

Electrical cardioversion: Eighty-four patients underwent electrical CV. Further sedation was given as needed. Incremental direct-current electrical shocks were delivered at 200, 300, and $360 \mathrm{~J}$ using cutaneous patch electrodes placed on the chest. All patients were successfully cardioverted.

One hundred and sixty-seven patients who had been successfully cardioverted to sinus rhythm were followed up for at least 6 months after CV. Patients were monitored for 24 hours after successful CV and started oral warfarin treatment. Dalteparin or UFH treatment was continued until the patient had reached therapeutic prothrombin values [international normalized ratio (INR) of 2.0 to 3.0]. All patients received warfarin for one month after CV and if AF recurred.

Statistical analysis: Results are expressed as mean \pm SD. Continuous variables were compared using Students' $t$ test. Quantitative variables were compared using the chi-square test. A $P$ value $<0.05$ was considered statistically significant.

\section{RESULTS}

Patient demographics: The two groups were comparable with respect to age, gender, duration of AF, etiological factors, and echocardiographic (TTE and TEE) findings. Mean AF duration was 80.2 \pm 65.3 days. Of these patients, 132 (77.6\%) had hypertension, $81(47.6 \%)$ coronary artery disease, $31(18.2 \%)$ diabetes mellitus type II, $53(31.2 \%)$ congestive heart failure, $19(11.2 \%)$ chronic obstructive lung disease, and 4 (2.4\%) prior cerebral events. The clinical and demographic parameters for 170 patients are summarized in Table I. Seven patients $(4.1 \%)$ had mitral valve prolapse and $19(11.2 \%)$ had mitral annulus calcification. The echocardiographic parameters are shown in Table II. There were no statistically significant differences between the LMWH and UFH groups.

Eighty-nine consecutive patients who underwent TTE and TEE were included in the LMWH group. In the LMWH group, 88 patients (44 medical CV, 44 electrical CV) were successfully cardioverted (98.9\%). CV (medical CV) was unsuccessful in 1 patient.

Eighty-one patients who underwent TTE and TEE were included in the UFH group. In the UFH group, 79 patients (39 medical CV, 40 electrical CV) 
Table I. Characterictics of the Low Molecular Weight Heparin Group and Standard Heparin Group

\begin{tabular}{lccc}
\hline & $\begin{array}{c}\text { LMWH group } \\
(n=89)\end{array}$ & $\begin{array}{c}\text { UFH group } \\
(n=81)\end{array}$ & $\begin{array}{c}P \\
\text { value }\end{array}$ \\
Female/Male & $46 / 43$ & $40 / 41$ & NS $(0.88)$ \\
Mean age (year) & $63.4 \pm 9.4$ & $61.9 \pm 10.2$ & NS (0.32) \\
Atrial fibrillation duration (day) & $80.9 \pm 66.5$ & $79.2 \pm 63.8$ & NS (0.87) \\
Hypertension & $67(75.3 \%)$ & $65(80.2 \%)$ & NS (0.55) \\
Coronary artery disease & $40(44.9 \%)$ & $41(50.6 \%)$ & NS $(0.56)$ \\
Diabetes mellitus & $16(18.0 \%)$ & $15(18.5 \%)$ & NS (0.91) \\
Heart failure & $28(31.5 \%)$ & $25(30.9 \%)$ & NS $(0.93)$ \\
Chronic obstructive pulmonary disease & $9(10.1 \%)$ & $10(12.3 \%)$ & NS (0.83) \\
Dilated cardiomyopathy & $5(5.6 \%)$ & $4(4.9 \%)$ & NS $(0.88)$ \\
Hypertrophic cardiomyopathy & $1(1.1 \%)$ & $1(1.2 \%)$ & NS (0.52) \\
Prior cerebrovascular event & $3(3.4 \%)$ & $1(1.2 \%)$ & NS (0.68) \\
Medical/Electrical cardioversion & $45 / 44$ & $41 / 40$ & NS (0.88) \\
Successful medical /electrical cardioversion & $44 / 44$ & $39 / 40$ & NS (0.94) \\
\hline
\end{tabular}

LMWH = Low molecular weight heparin; UFH = Unfractionated heparin; NS = Nonsignificant.

Table II. Echocardiographic Parameters of the Low Molecular Weight Heparin Group and Standard Heparin Group

\begin{tabular}{lccc}
\hline & $\begin{array}{c}\text { LMWH group } \\
(n=89)\end{array}$ & $\begin{array}{c}\text { UFH group } \\
(n=81)\end{array}$ & $\begin{array}{c}P \\
\text { value }\end{array}$ \\
Mitral valve prolapse & $4(4.5 \%)$ & $3(3.7 \%)$ & NS $(0.90)$ \\
Mitral annulus calcification & $9(10.1 \%)$ & $10(12.3 \%)$ & NS $(0.83)$ \\
Mitral regurgitation & $10(11.2 \%)$ & $11(13.6 \%)$ & NS $(0.82)$ \\
Mean left atrium diameter $(\mathrm{cm})$ & $4.2 \pm 0.5$ & $4.3 \pm 0.6$ & NS $(0.24)$ \\
Left atrial diameter $\leq 4.0 \mathrm{~cm}$ & $35(39.3 \%)$ & $32(39.5 \%)$ & NS $(0.89)$ \\
Mean left ventricle diameter $(\mathrm{cm})$ & $5.8 \pm 0.6$ & $5.7 \pm 0.8$ & NS $(0.36)$ \\
Left ventricular ejection fraction $<\% 55$ & $30(33.7 \%)$ & $27(33.3 \%)$ & NS $(0.91)$ \\
Spontaneous echo contrast & $37(41.6 \%)$ & $34(42.0 \%)$ & NS $(0.92)$ \\
\hline
\end{tabular}

LMWH = Low molecular weight heparin; UFH = Unfractionated heparin; NS = Nonsignificant.

were successfully cardioverted to sinus rhythm (97.5\%). CV was unsuccessful in 2 patients.

After one month follow-up, sinus rhythm was maintained in $88.2 \%$ of the patients in the LMWH group compared to $85.7 \%$ in the UFH group. Ninety-eight $(58.7 \%)$ patients were still in sinus rhythm 6 months after CV. There was no significant difference in the proportion of patients in whom normal sinus rhythm had been maintained between the two groups $(61.6 \%$ in the LMWH group and $55.6 \%$ in the UFH group).

None of the patients in either group experienced thromboembolic events during the four weeks after CV. 


\section{DISCUSSION}

Restoration of sinus rhythm provides numerous physiological benefits, ${ }^{12)}$ but as with most new therapies, direct-current $\mathrm{CV}$ also has adverse outcomes, including catastropic thromboembolism in up to 6.3 percent of patients who were not receiving anticoagulant therapy. ${ }^{13)}$ Nonrandomized studies where warfarin therapy was given for 3 to 4 weeks before $\mathrm{CV}$ led to an 80 percent reduction in $\mathrm{CV}$-related thromboembolism, ${ }^{14)}$ providing the rationale for the use of this strategy for nearly three decades.

Until recently, most clinicians assumed that thromboembolism at the time of $\mathrm{CV}$ was a result of the migration of left atrial thrombi that were already present. ${ }^{15}$ ) Despite the remarkable visualization of cardiac structures with TTE, it was quickly recognized that this technique was inadequate for the identification of atrial thrombi, especially thrombi that were confined to the atrial appendages. ${ }^{16}$ Promising initial reports of the potential of TEE for facilitating early $\mathrm{CV}^{4)}$ were followed by reports of adverse events, ${ }^{17)}$ leaving clinicians without a sense of clear direction. Since conventional therapy was associated with a minimal rate of clinical thromboembolism (less than 1\%), many argued that no change should be made. For patients in $\mathrm{AF}$ who require urgent $\mathrm{CV}$ and patients who may benefit from immediate elective $\mathrm{CV}$, intravenous UFH had been the anticoagulant of choice. ${ }^{18,19)}$ Intravenous UFH provides rapid antithrombotic therapy and effective bridge therapy pending the achievement of a therapeutic INR of 2.0 to 3.0 from oral warfarin. In more than 1300 patients previously studied by North American and European investigators, there was only 1 documented embolic event in cases involving treatment guided by TEE with short-term anticoagulation..$^{20,21-23)}$ The TEE-guided approach may reduce the time to CV compared with the conventional approach, as shown by the ACUTE Pilot Study (4.8 versus 0.6 weeks, $P<$ 0.01). ${ }^{3)}$ Earlier CV may decrease the risk of bleeding compared with a more prolonged conventional approach. In general, the duration of pre-CV antithrombotic therapy is reduced by $88 \%$ for patients undergoing early TEE-guided CV, either as an inpatient or outpatient, compared with the conventional non-TEE approach. ${ }^{3)}$

We studied 252 patients with nonvalvular AF of more than 2 days and less than 1 year. ${ }^{24)}$ The aim of this study was to determine the feasibility and safety of TEE guided CV with short-term anticoagulation in patients with nonvalvular AF. Patients in whom TEE revealed no atrial or ventricular thrombi underwent pharmacological or electrical CV under IV UFH therapy followed by warfarin for 1 month. There were no thromboembolic events.

The most recent study was the Assessment of Cardioversion Using Transesophageal Echocardiography (ACUTE) Multicenter Study. ${ }^{4)}$ The purpose of the 
ACUTE study was to compare a conventional anticoagulant therapy with the strategy of using TEE to guide short-term anticoagulant therapy in patients with AF of more than two days duration. In the TEE group intravenous UFH (target APTT, 1.5 to 2.5 times the control value) was used. Both treatment strategies (TEE guided treatment or conventional treatment) in this study resulted in low rates of embolism and there was no statistically significant difference between the two groups in the rate of such events [five events in the TEE group $10.8 \%$; $95 \%$ confidence interval, 0 to 1.5$)$ and three events in the conventional treatment group $(0.5 \%$; $95 \%$ confidence interval, 0 to $1.1 \%), P=0.50]$. The ACUTE Multicenter Study showed that the composite major or minor bleeding complication rate for the conventional approach was significantly more than the TEE guided approach, and it suggested that the strategy of using TEE to guide anticoagulant therapy for AF was both feasible and safe.

With the use of a decision analysis model, Seto, et $a l^{25)}$ showed that the TEE-guided UFH approach to early CV, without TTE, is a reasonable cost-saving alternative to the conventional $\mathrm{UFH}$ approach to $\mathrm{CV}$ for patients admitted to the hospital for AF.

An approach combining short-term LMWH and TEE has been proposed as an alternative for the anticoagulation management of patients in AF who undergo $\mathrm{CV}^{26)}$ In patients recommended for immediate CV, TEE may serve to screen patients for atrial thrombi, and LMWH may provide an economically attractive alternative to $\mathrm{UFH}^{26)}$ Because its anticoagulant response is predictable, ${ }^{27)}$ in hospital therapy and the monitoring of APTT are not required.

Roijer, et $a^{28)}$ studied 242 patients with $\mathrm{AF}$ and a low thromboembolic risk for CV. After the TEE examination, patients who were eligible for immediate CV were anticoagulated with LMWH (dalteparin) subcutaneously, together with warfarin prior to $\mathrm{CV}$. Thromboembolic risk was low if there were no thrombi and no echo spontaneous contrast and the outflow velocity of the left atrial appendage was greater than $0.25 \mathrm{~ms}^{-1}$ on TEE. Based on the TEE findings the patients were divided into two groups; an immediate $\mathrm{CV}$ group and a conventional warfarin treatment group. No thromboembolic events occurred at or after $\mathrm{CV}$ in any of the patients.

Murray, et $a l^{29)}$ studied 1868 patients with AF for CV. They reported an alternative clinical management strategy and cost analysis model was present for patients with AF more than 2 days duration who might benefit from immediate $\mathrm{CV}$ with self-administered LMWH (enoxaparin), after a negative TEE screening for thrombus.

The study design utilized in the present trial by Murray, et al allowed direct comparison to be made between LMWH and UFH in the treatment of nonvalvular AF (AF duration > 2 days). We found that during four weeks of study, there 
was no significant difference between the two treatment groups in the immediate success of CV (medical and electrical). At six months there was no significant difference between the two groups in the proportion of patients in whom normal sinus rhythm had been maintained $(61.6 \%$ in the LMWH group and $55.6 \%$ in the UFH group). No difference in the incidence of thromboembolic events after $\mathrm{CV}$ was seen between LMWH and UFH. These authors have underlined the potential advantages of the TEE guided LMWH approach to CV for patients in AF who undergo $\mathrm{CV}{ }^{29)}$

\section{CONCLUSION}

TEE guided early CV in conjunction with short-term LMWH (Dalteparin) treatment is as safe as standard IV heparin for the prevention of thromboembolic events after $\mathrm{CV}$. These results have important clinical and economic implications for the antithrombic management of patients with AF undergoing TEE guided CV.

\section{REFERENCES}

1. Lip GHY. Paroxysmal atrial fibrillation. In : Lip G, editor. Atrial Fibrillation in Clinical Practice. First edition, London, Martin Dunitz Ltd, 2001: 137-47.

2. Laupacis A, Alberts G, Dunn M, Feinberg W. Antithrombotic therapy in atrial fibrillation. Chest 1992; 102: 426-31.

3. Klein AL, Grimm RA, Black IW, et al. Cardioversion guided by transesophageal echocardiography : the ACUTE Pilot Study - a randomized, controlled trial. Assessment of cardioversion using transesophageal echocardiography. Ann Intern Med 1997; 126: 200-9.

4. Klein AL, Grimm RA, Murray DOR, et al. Use of transesophageal echocardiography to guide cardioversion in patients with atrial fibrillation. N Engl J Med 2001; 344: 1411-20.

5. Hirsh J, Levine MN. Low molecular weight heparin. Blood 1992; 79: 1-17.

6. Warkentin TE, Levine MN, Hirsh J, et al. Heparin-induced thrombocytopenia in patients treated with low molecular weight heparin or unfractionated heparin. N Engl J Med 1995; 332: 1330-5.

7. Weitz JI. Low-molecular weight heparins. N Engl J Med 1997; 337: 688-98.

8. Hyers TM, Agnelli G, Hull RD, et al. Antithrombotic therapy for venous thromboembolic disease. Chest 1998; 114: 516S-578S.

9. Bjerkelund CJ, Orning OM. The efficacy of anticoagulant therapy in preventing embolism related to D.C. electrical conversion of atrial fibrillation. Am J Cardiol 1969; 23: 208-16.

10. Spinler SA, Nawarskass JJ. Low-molecular-weight heparins for acute coronary syndromes. Ann Pharmacother 1998; 32: 103-10.

11. Fatkin D, Kuchar DL, Thorburn CW, Feneley MP. Transesophageal echocardiography before and during current cardioversion of atrial fibrillation : evidence for "atrial stunning" as a mechanism of thromboembolic complications. J Am Coll Cardiol 1994; 23: 307-16.

12. Black IW, Fatkin D, Sagar KB, et al. Exclusion of atrial thrombus by transesophageal echocardiography does not preclude embolism after cardioversion of atrial fibrillation: A multicenter study. Circulation 1994; 89: 2509-13.

13. Arnold AZ, Mick MJ, Mazurek RP, Loop FD, Trohman RG. Role of prophylactic anticoagulation for direct current cardioversion in patients with atrial fibrillation or atrial flutter. J Am Coll Cardiol 1992; 19: 581-5. 
14. Leizorovicz A, Simonneau G, Decousus H, Boissel JP. Comparison of efficacy and safety of low molecular weight heparins and unfractionated heparin in initial treatment of deep venous thrombosis : a meta-analysis. BJM 1994; 309: 299-304.

15. Goldman MJ. The management of chronic atrial fibrillation : indications for and method of conversion to sinus rhythm. Prog Cardiovasc Dis 1960; 2: 465-79.

16. Aschenberg W, Schülter M, Kremer P, Schroder E, Siglow V, Bleifeld W. Transesophageal two-dimensional echocardiography for the detection of left atrial appendage thrombus. J Am Coll Cardiol 1986; 7: 163-6.

17. Pritchett ELC. Management of atrial fibrillation. N Engl J Med 1992; 326: 1264-71.

18. Mayet J, Wasan B, Sutton GC. Cardioversion of atrial arrhytmias : audit of anticoagulation management. J Royal Coll Phys London 1997; 31: 313-6.

19. Schlicht JR, Davis RC, Naqi K, Cooper W, Rao BV. Physician practices regarding anticoagulation and cardioversion of atrial fibrillation. Arch Intern Med 1996; 156: 290-4.

20. Manning WJ, Silverman DI, Gordon SPE, Krumholz HM, Douglas PS. Cardioversion from atrial fibrillation without prolonged anticoagulation with use of transesophageal echocardiography to exclude the presence of atrial thrombi. N Engl J Med 1993; 328: 750-5.

21. Manning WJ, Silverman DI, Keighley CS, Oettgen P, Douglas PS. Transesophageal echocardiography facilitated early cardioversion from atrial fibrillation using short-term anticoagulation : final results of a prospective 4.5 year study. J Am Coll Cardiol 1995; 25: 1354-61.

22. Stoddard MF, Dawkins PR, Prince CR, Longaker RA. Transesophageal echocardiographic guidence of cardioversion in patients with atrial fibrillation. Am Heart J 1995; 129: 1204-15.

23. Corrado G, Tadeo G, Berretta S, et al. Atrial thrombi resolution after prolonged anticoagulation in patients with atrial fibrillation. Chest 1999; 115: 140-3.

24. Yigit Z, Küçükoglu S, Sansoy V, et al. TEE-guided cardioversion is feasible and safe in patients with nonvalvular atrial fibrillation. Arch Turkish Soc Cardiol 1998; 26: 348-53.

25. Seto TB, Taira DA, Tsevat J, Manning WJ. Cost-effectiveness of transesophageal echocardiographic-guided cardioversion: a decision analytic model for patients admitted to the hospital with atrial fibrillation. J Am Coll Cardiol 1997; 29: 122-30.

26. Murray RD, Jasper SE, Goodman A, et al. Cost analysis model of low molecular weight heparin for antithrombotic therapy in patients undergoing TEE-guided cardioversion from atrial fibrillation (Abstract). J Am Coll Cardiol 1999; 33: 294A.

27. Noble S, Peters DH, Goa KL. Enoxaparin: a reappraisal of its pharmacology and clinical applications in the prevention and treatment of thromboembolic disease. Drugs 1995; 49: 388-410.

28. Roijer A, Eskilsson J, Olsson B. Transesophageal echocardiography-guided cardioversion of atrial fibrillation or flutter : selection of a low-risk group for immediate cardioversion. Eur Heart J 2000; 21: 837-47.

29. Murray RD, Deitcher SR, Shah A, et al. Potential clinical efficacy and cost benefit of a transesophageal echocardiography-guided low-molecular-weight heparin (enoxaparin) approach to antithrombotic therapy in patients undergoing immediate cardioversion from atrial fibrillation. J Am Soc Echocardiogr 2001; 14: 200-8. 